\title{
UTILISATION DE LEVURE DE DISTILLERIE DANS L'ALIMENTATION DES VOLAILLES \\ PAR
}

\section{P. CHARLET \\ G. CHARLET-LERY \\ A. M. LEROY}

Laboratoire de Recherches de Zootechnie de l'Institut National Agronomique, Paris

\section{PLAN DU MEMOIRE}

I. - Utilisation de la levure de distillerie dans l'alimentation de poules pondeuses et reproductrices.

II. - Utilisation de la levure de distillerie dans l'alimentation des poussins.

III. - Comparaison des levures de distillerie et de brasserie pour l'alimentation des poussins.

\section{I. - LUTILISATION DE LA LEVURE DE DISTILLERIE DANS l'Alimentation des poules pondeuses et RePRoductrices}

\section{Introduction}

L'utilisation des différentes levures dans l'alimentation des volailles a déjà fait l'objet de nombreuses études, mais les auteurs ont le plus souvent envisagé les levures comme source du complexe vitaminique $B$, et récemment'de l' "animal protein factor " et, de ce fait, ils recommandent l'introduction d'un pourcentage toujours relativement faible de levure dans les rations pour volailles.

C'est le point de vue de Synold et Coll (Io) et de Novak et Coli (9). Milíigan (7) étudie la supplémentation en riboflavine d'une ration de base pour poussins, déficiente en cet élément ; riboflavine synthétique, mycélium desséché de pénicillium, levure de brasserie ont tous des actions nettement favorables sur la croissance depuis la naissance jusqu'à 9 semaines. Le remplacement de 2 p. Ioo d'orge par 2 p. Ioo de culture de levure vivante chez les poussins en croissance joue très légèrement en faveur de cette dernière (2). Les 
mêmes cultures semblent avoir une action plus nette sur les coquelets que sur les poulettes (3). 'TiTus (II), WaLiIs (I2) recommandent des doses analogues: 2 à 3 p. Ioo pour des poussins au démarrage, 2 à 4 p. Ioo pour les poulets en croissance et pour les pondeuses dans l'aliment complet. MARCQ et LAHAYE (8) réduisent encore ces normes et conseillent I I/2 p. Ioo de levure pour les poussins.

Une étude sur les levures extraites à partir des cuves sulfitées des pulpes et des pâtes à papier (I) montre que ces levures seraient nettement moins efficaces pour la croissance des jeunes - $20 \mathrm{p}$. Ioo - que le tourteau de soja lorsqu'jls sont donnés l'un et l'autre conme seule source de matières azotées. Mais la levure se révèle intéressante pour la ponte et contient 1'A. P. F. nécessaire pour l'éclosion des oufs et la croissance des jeunes.

lans son travail, M. C. Grviss (5) envisage la levure comme une source de matières azotées et en distribue de fortes doses. Il ne constate pas d'accidents chez les poussins avec des régimes contenant i2 p. Ioo de torula ou de levure de distillerie. Pour les poules New-Hampshire, 5 p. Ioo de levure dans le régime n'augmente pas le pouvoir d'éclosion mais permet la naissance de poussins plus vigoureux. Pour lui, la levure, quelle que soit sa présentation, est avantageusement utilisée dans les régimes pour poussins, à la condition de ne pas être distribuée en trop grande quantité aux animaux trop jeunes; donnée aux adultes elle donne satisfaction pour la ponte mais n'angmente pas le pouvoir d'éclosion; en cela, elle reste inférieure à la farine de poisson.

Ce dernier rapport pose d'intéressants problèmes. L'un des inconvénients des farines animales (viande, poisson ou sang) est leur difficulté de conservation. La levure, par contre, se conserve extrêmement bien et pendant un temps fort long. I)es levures de brasserie ou de distillerie gardées sans précautions spéciales, d'octobre I 949 à mai $\mathrm{I} 95 \mathrm{I}$, ont vu leur acidité totale varier de 2,0 à 2,4 et 2,5 p. Ioo. Les levures de distillerie sont, d'après leur prix de revient, nettement plus économiques à utiliser que les levures de brasserie, déjà bien connues des éleveurs. Malheureusement, en France, la récupération des levures de distillerie a lieu suivant des procédés relativenent différents d'une usine à l'autre et, si certaines levures donnent de bons résultats, dans d'autres cas, des accidents de pseudo-rachitisme ont été signalés chez les poussins par des éleveurs privés.

Dans le cadre métropolitain français, la possibilité d'utiliser de fortes teneurs de levure de distillerie dans les aliments pour volailles présenterait l'avantage de mettre sur le marché un produit de haute valeur biologique, pouvant remplacer à la fois les farines animales - de conservation délicate ainsi qu'un certain nombre de tourteaux d'importation.

Nous avons donc voulu dans les expériences suivantes, étudier les possibilités d'emploi de la levure de distillerie comme source de matières azotées, tant pour la ponte et la reproduction, que pour la croissance des jeunes.

L'expérience a été conduite à la station avicole de Saint-Leu-la-Forêt (Laboratoire de recherches de la Chaire de Zootechnie de l'I. N. A.). 


\section{Étude de la ponte et des phénomènes de reproduction}

L'expérience a porté sur l'année de ponte allant du $\mathrm{I}^{\mathrm{er}}$ octobre 1949 au I er octobre I950.

Parmi les poulettes Gâtinaises de la Station avicole, nous avons constitué 2 groupes homogènes quant à leur poids et à leur origine. La production a été contrôlée au nid trappe et la consommation a été surveillée en qualité et en quantité.

Chaque lot comprenait 8 poules à leur première année de ponte et 2 poules à leur $2^{\mathrm{e}}$ année de ponte.

I'alimentation avait pour base un aliment concentré dont la formule et l'analyse chimique étaient les suivantes :
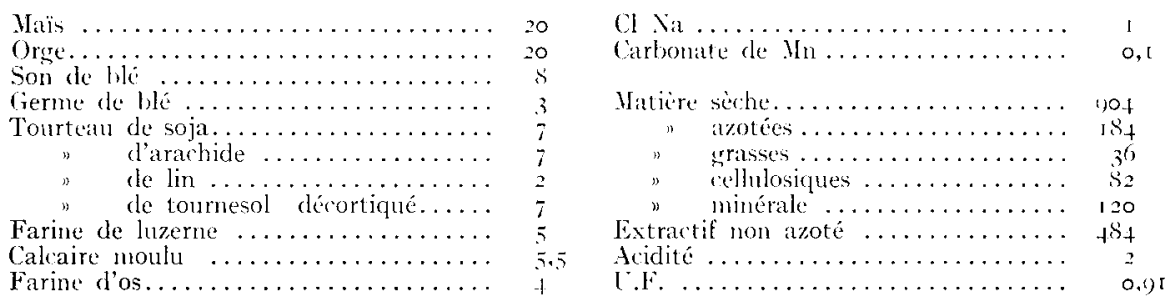

Cet aliment concentré de base était complété dans le lot I par I2 p. Ioo de levure de distillerie, dans le lot II (lot témoin) par 6 p. Ioo de farine de viande et 4 p. roo de levure de distillerie. L exactement équiazotés : $215 \mathrm{~g}$ de matières azotées brutes an $\mathrm{kg}$.

La levure de distillerie et la farine de viande correspondaient aux analyses ci-dessous.

\begin{tabular}{|c|c|c|}
\hline & $\begin{array}{l}\text { Levure de } \\
\text { distillerie. } \\
\text { - }\end{array}$ & $\begin{array}{l}\text { Farine de } \\
\text { viande. }\end{array}$ \\
\hline Matière sieche ................... & 892 & (1) 26 \\
\hline Matiures azoties .................... & +42 & 536 \\
\hline p grasses $\ldots \ldots \ldots \ldots \ldots \ldots \ldots \ldots$ & 29 & 220 \\
\hline$r \quad$ minćrates $\ldots \ldots \ldots \ldots \ldots \ldots \ldots \ldots$ & 50 & 224 \\
\hline Extractif non azoté.............. & $37 \mathrm{I}$ & - \\
\hline Acidite $\ldots \ldots \ldots \ldots \ldots \ldots \ldots \ldots \ldots$ & 2 & 4.8 \\
\hline 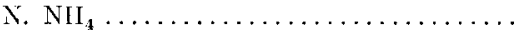 & 一 & 0,85 \\
\hline
\end{tabular}

Ces mélanges étaient distribués à volonté, les quantités consommées étaient contrôlées exactement, ce qui permettait de compléter la ration par $30 \mathrm{p}$. Ioo d'avoine. La ration totale apportait is p. Ioo de matières azotées brutes au $\mathrm{kg}$ - et comprenait dans le lot I: 9,25 p. Ioo de levure de distillerie, dans le lot II : 3, I p. roo.

Les vitamines $A$ et $D$ étaient apportées sous forme d'huile de foie de poisson à la dose de ro o00 $u$. $i$. de vitamine $\mathrm{A}$ et $\mathrm{x} 300 u$. $i$. de vitamine $\mathrm{D}_{3}$ par kg de pâtée ; l'huile était mélangée à la pâtée immédiatement avant la distribution de celle-ci. Les animaux recevaient une partie de l'avoine sous forme germée. 


\section{Io Etat de santé}

Nous avons eu à enregistrer quelques décès parmi les volailles en expérience par suite d'une forte attaque de coccidiose en octobre et novembre : 2 animaux dans le lot I et un dans le lot II ont été, pour cette raison, remplacés. Sur les autres animaux, nous avons eu seulement, avec des chutes de poids considérables, des arrêts de ponte ou des entrées en ponte tardive qui n'ont pas permis d'obtenir les productions attendues.

Une poule du lot I est morte d'une occlusion intestinale au cours de l'année.

\section{$2^{\circ}$ Poids}

Le poids moyen des animaux s'est maintenu en moyenne, pour toute la durée de l'étude, légèrement au-dessus de $2 \mathrm{~kg}: 2,0$ IO $\mathrm{kg}$ pour le lot I; 2,050 kg pour le lot II.

\section{$3^{\circ}$ Ponte}

Le tableau suivant résume les résultats obtenus :

'Tableau I

\begin{tabular}{|c|c|c|c|c|c|}
\hline & $\begin{array}{c}\text { Nombre total } \\
\text { d'oufs }\end{array}$ & $\begin{array}{l}\text { Nombre } \\
\text { moyen } \\
\text { par poule }\end{array}$ & $\begin{array}{l}\text { Poids total } \\
\text { en kg. }\end{array}$ & $\begin{array}{l}\text { Poids moyen } \\
\text { d'un œuf } \\
\text { en g. }\end{array}$ & $\begin{array}{l}\text { Poids moyen } \\
\text { d'œuf par } \\
\text { poule et } \\
\text { par jour } \\
\text { en g. }\end{array}$ \\
\hline $\begin{array}{l}\text { Lot I } \\
\text { Poules de I ere année }(7) .\end{array}$ & & & & & \\
\hline jusqu'au I $^{\text {er }}$ octobre.... & $8+14$ & 20,6 & 49,995 & 59,2 & 19,57 \\
\hline $\begin{array}{l}\text { jusqu'au I } \text { I }^{\text {r novembre... }} \\
\text { Poules de I ere et } 2^{\mathrm{e}} \text { années }(9)\end{array}$ & 922 & 131,7 & 54,415 & 59,0 & 19,63 \\
\hline $\begin{array}{l}\text { jusqu'au } \text { I }^{\text {er }} \text { ortobre } . . . . \\
\text { jusqu'au } \\
\text { I }^{\text {er }} \text { novembre.... }\end{array}$ & $\begin{array}{r}991 \\
\text { I } 069\end{array}$ & $\begin{array}{l}\text { I I } 0, \text { I } \\
\text { I } 8,8\end{array}$ & 59,272 & $\begin{array}{l}59,8 \\
59,7\end{array}$ & 18,04 \\
\hline $\begin{array}{l}\text { Lot } I I \\
\text { Poules de I }{ }^{\text {ere }} \text { année }(8)\end{array}$ & & & & & \\
\hline jusquau ${ }^{\text {er }}$ octobre ..... & 892 & I I 1,5 & $5^{1,255}$ & 57,6 & I 7,55 \\
\hline $\begin{array}{l}\text { Jusqu'au I } \text { er }^{\text {jovembre... }} \\
\text { l'oules de I }{ }^{\text {ere }} \text { et } 2^{\mathbf{e}} \text { ancée (Io) }\end{array}$ & 944 & I I 8,0 & 54,450 & 57,6 & 17,18 \\
\hline jusqu'au I er octobre.... & I 080 & 108,0 & 62,095 & 57,5 & 17,01 \\
\hline jusqu'au I $^{\text {er }}$ novembre... & 1142 & $1 I_{4}, 2$ & 一 & 57,5 & \\
\hline
\end{tabular}

Par suite de l'entrée en ponte tardive, un certain nombre de poules ont continué à pondre en octobre -5 sur 7 dans le lot expérimental, 4 sur 8 dans le lot témoin - et nous avons pensé pouvoir en tenir compte bien qu'à partir du I er octobre tous les animaux aient été soumis à la même alimentation.

Ces valeurs sont toutes légèrement en faveur du lot expérimental qui recevait I2 p. Ioo de levure, mais, par suite de la dispersion des résultats, les différences entre ces données ne sont pas significatives. On ne peut donc pas conclure à une amélioration de la ponte due à la consommation d'une quantité élevée de levure de distillerie. 


\section{$4^{0}$ Consommation}

La distribution quantitative a été contrôlée avec grand soin du Ier novembre au 30 juin et pendant cette période les animaux ont absorbé en moyenne:

Dans le lot I par animal (poule et coq) et par jour,

95,5 g de mélange,

$3 \mathbf{I}, 8 \mathrm{~g}$ d'avoine.

Dans le lot II par animal (poule et coq) et par jour,

I $6,5 \mathrm{~g}$ de mélange,

38,9 g d'avoine.

Dans le lot I, les animaux ont reçu, pendant cette période, un total de 280 unités fourragères lorsque les besoins théoriques s'élevaient à 268 unités. Par contre, les animaux du lot II ont reçu, pendant la même période, 372 unités fourragères au lieu de 320 qui correspondaient à leurs besoins théoriques.

Si le contrôle de la distribution des aliments peut s'effectuer aisément, celui de la consommation réelle ne peut pas s'obtenir avec une précision extrême car il y a toujours un léger pourcentage de perte dû au gaspillage des animaux et parfois, en hiver, des déprédations commises par des rongeurs, ce qui ne permet pas d'attribuer une valeur absolue aux données ci-dessus. Cependant, il semble permis de dire que le lot expérimental a nettement mieux utilisé la ration mise à sa disposition que le lot témoin.

\section{$5^{\circ}$ Phénomènes de reproduction}

Les résultats d'incubation ont été identiques dans les 2 lots, car les pourcentages d'œufs non féconds ont été de o p. roo dans le lot expérimental et de 7 p. Ioo dans le lot témoin et les pourcentages d'éclosion par rapport au nombre total d'œufs mis à couver, ont été respectivement de 76 et $77 \mathrm{p}$. Ioo.

Les poussins, quelle que soit leur origine, se sont montrés également vigoureux et nous n'avons enregistré aucun décès durant les 2 premiers mois où la croissance s'est montrée constante et régulière.

\section{II. - UTILISATION DE LA LEVURE DE DISTILLERIE DANS L'ALIMENTATION DES POUSSINS}

\section{Étude de la croissance des jeunes}

Une soixantaine de poussins Gâtinais nés le 6 juin I950 ont été séparés en deux lots identiques d'après leur poids et mis en batterie. Ces animaux étaient issus des poules placées soit dans le lot témoin, soit dans le lot expérimental. Comme nous avions constaté précédemment 1'identité de croissance des poussins issus de ces deux lots, nous n'avons pas jugé utile de tenir compte de l'origine des poussins pour leur répartition entre les deux lots.

Le lot témoin a reçu une farine concentrée apportant $192 \mathrm{~g}$ de protéines 
brutes au $\mathrm{kg}$. Cette farine contenait $9 \mathrm{p}$. Ioo d'un mélange de farine de sang, viande et poisson qui furent remplacés dans le lot d'expérience par I4 p. roo de levure de distillerie sans aucun apport supplémentaire de matières minérales. Le reste de la ration (céréales, son fin, farine de luzerne et tourteaux) apportait en outre $3 \mathrm{p}$. Ioo de lactalbumine.

Le tableau suivant donne les résultats des analyses des divers produits ntilisés au cours de cette expérience :

\begin{tabular}{|c|c|c|c|c|c|c|}
\hline & $\begin{array}{l}\text { Matière } \\
\text { sèche }\end{array}$ & $\begin{array}{l}\text { Matières } \\
\text { azotées }\end{array}$ & $\begin{array}{l}\text { Matiere } \\
\text { grasse }\end{array}$ & $\begin{array}{l}\text { Matières } \\
\text { cellulos. }\end{array}$ & $\begin{array}{l}\text { Matières } \\
\text { Minérales }\end{array}$ & $\begin{array}{l}\text { Extractifs } \\
\text { non azotés }\end{array}$ \\
\hline Mélange complet témoin... & 880 & 192 & 56 & 49 & 82 & 502 \\
\hline Mélange témoin sans farine & & & & & & \\
\hline Farine d'origine animale... & $\begin{array}{l}803 \\
879\end{array}$ & 583 & 417 & 52 & $\begin{array}{ll}7 \mathrm{~T} \\
\mathrm{I} 44\end{array}$ & $53^{2}$ \\
\hline Levure de distillerie ...... & 892 & 442 & 29 & - & 50 & $37 \mathrm{I}$ \\
\hline
\end{tabular}

Les deux lots recevaient des rations ayant pratiquement la même richesse en azote : $192 \mathrm{~g}$ de matières azotées brutes par $\mathrm{kg}$ dans le lot témoin contre I99 $\mathrm{g}$ dans le lot à la levure. Ce dernier était moins riche en matières minérales : $68 \mathrm{~g}$ contre $82 \mathrm{~g}$ et un peu moins riche en matières cellulosiques : $45 \mathrm{~g}$ contre $49 \mathrm{~g}$.

Dès la troisième semaine les animaux ont reçu de petites quantités d'avoine germée ; ces quantités ont augmenté progressivement à mesure que les animaux avançaient en âge.

Les 2 lots ont reçu les mêmes quantités de vitamines $A$ et $\mathrm{D}_{3}$ sous forme d'huile de foie de morue mélangée à la pâtée juste avant sa distribution.

A 60 jours, les poulets avaient atteint les poids suivants :

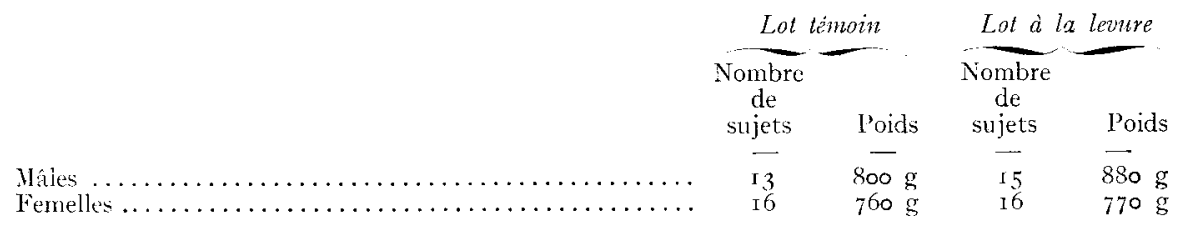

L.es croissances sont identiques dans le cas des femelles en faveur du lot à la levure dans le cas des coquelets : les résultats dans ce cas sont significativement différents (fig. I).

Nous avons également observé un emplumage nettement plus rapide et plus homogène dans le lot à la levure.

Dans une autre couvée, née au début de juillet, 38 poussins ont reçu un régime contenant $\mathrm{I} 4 \mathrm{p}$. Ioo de levure de distillerie et nous avons constaté des croissances normales et régulières. Leur poids à 60 jours s'élevait à : 
Une vingtaine de poulettes provenant de ces deux expériences ont été gardées pour observer leur entrée en ponte qui a lieu de la même façon pour les deux groupes en décembre et janvier pour les premiers, en janvier et février pour les seconds. Les poulettes étaient dans les deux cas âgées de 6 à 7 mois.

Ces expériences montrent que la levure de distillerie peut être utilisée comme seule source de matières azotées sans qu'il soit fait appel à des produits d'origine animale - exception faite des sous-produits de laiterie - et donne des résultats égaux ou supérieurs à un mélange de farine de viande, de poisson et de sang.

\section{III. - COMPNRAISON DES LEVURES DE DISTILLERIE ET DE BRASSERIE POUR L'ALIMENTATION DES POUSSINS}

Au cours du printemps I95I, nous avons comparé les valeurs des levures de brasserie et de distillerie pour la croissance des poussins.

I26 poussins Gâtinais ont été divisés à leur naissance en deux lots identiques quant à leur poids et leur origine. Ils ont reçu durant toute l'expérience des régimes semblables qui ne se différenciaient que par la présence de levure de distillerie ou de levure de brasserie. Nous avons porté le taux de levures à ro p. Ioo.

La ration totale était ainsi composée :

Farine complete du commerce $\ldots . . . \ldots \ldots \ldots \ldots \ldots \ldots$. s. so $\%$

I.evure de distillerie ou levure de brasserie ............... to $\%$

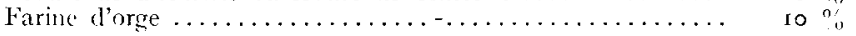

La farine du commerce répondait aux caractéristiques suivantes :

\section{Composition :}

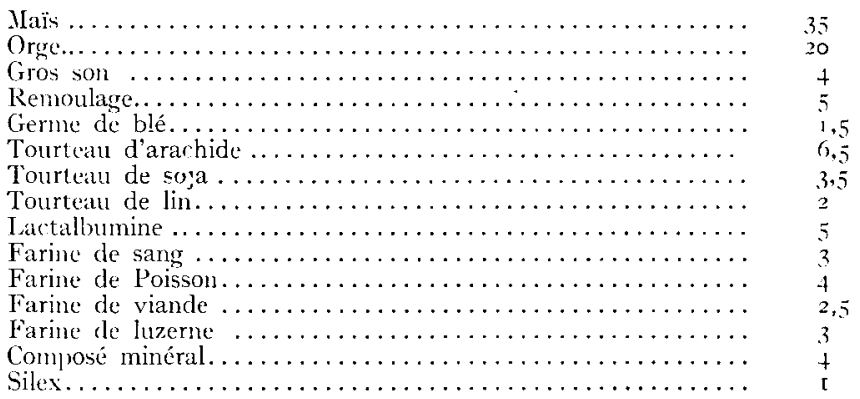

Analyse :

IItmidite $\ldots \ldots \ldots \ldots \ldots \ldots \ldots \ldots \ldots \ldots \ldots \ldots \ldots \ldots \ldots \ldots$ I

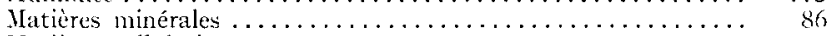

Matieres cellulosiques.......................... 39

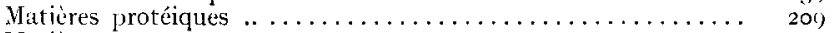

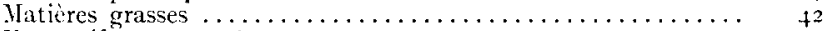

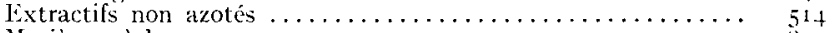

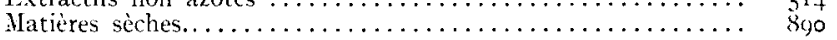

Les levures contenaient respectivement $442 \mathrm{~g}$ l et $486 \mathrm{~g}$ de matières protéiques par $\mathrm{kg}$; l'orge 78 ; ce qui portait les teneurs des rations en matières 
protéïques brutes à 2 I9 et 223 p. mille, teneurs que l'on peut considérer comme pratiquement équivalentes.

Les rations ont été supplémentées en vitamine $A$ et $D_{3}$ suivant les normes courantes.

A partir de la $3^{\mathbf{e}}$ semaine, les poussins ont reçu de petites quantités d'avoine qui ont été augmentées progressivement.

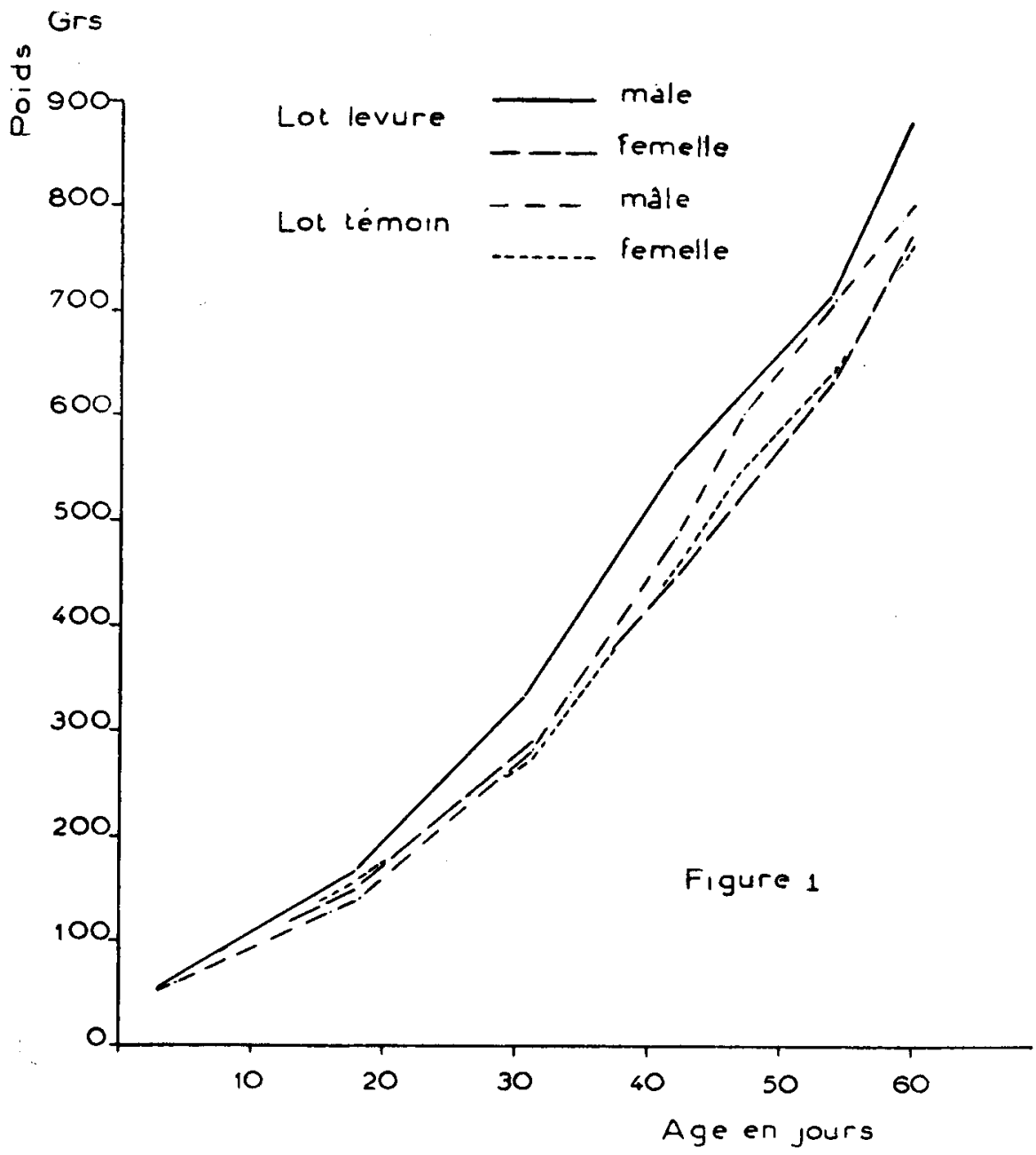

Six poussins sont morts accidentellement au début de l'expérience.

A 60 jours, les poulets pesaient : 
Les courbes de poids ci-jointes (fig. 2) montrent la régularité de croissance des deux lots qui ne se différencient que dans les derniers jours, mais cette différence n'est pas significative. Ce qui permet de conclure que la levure de

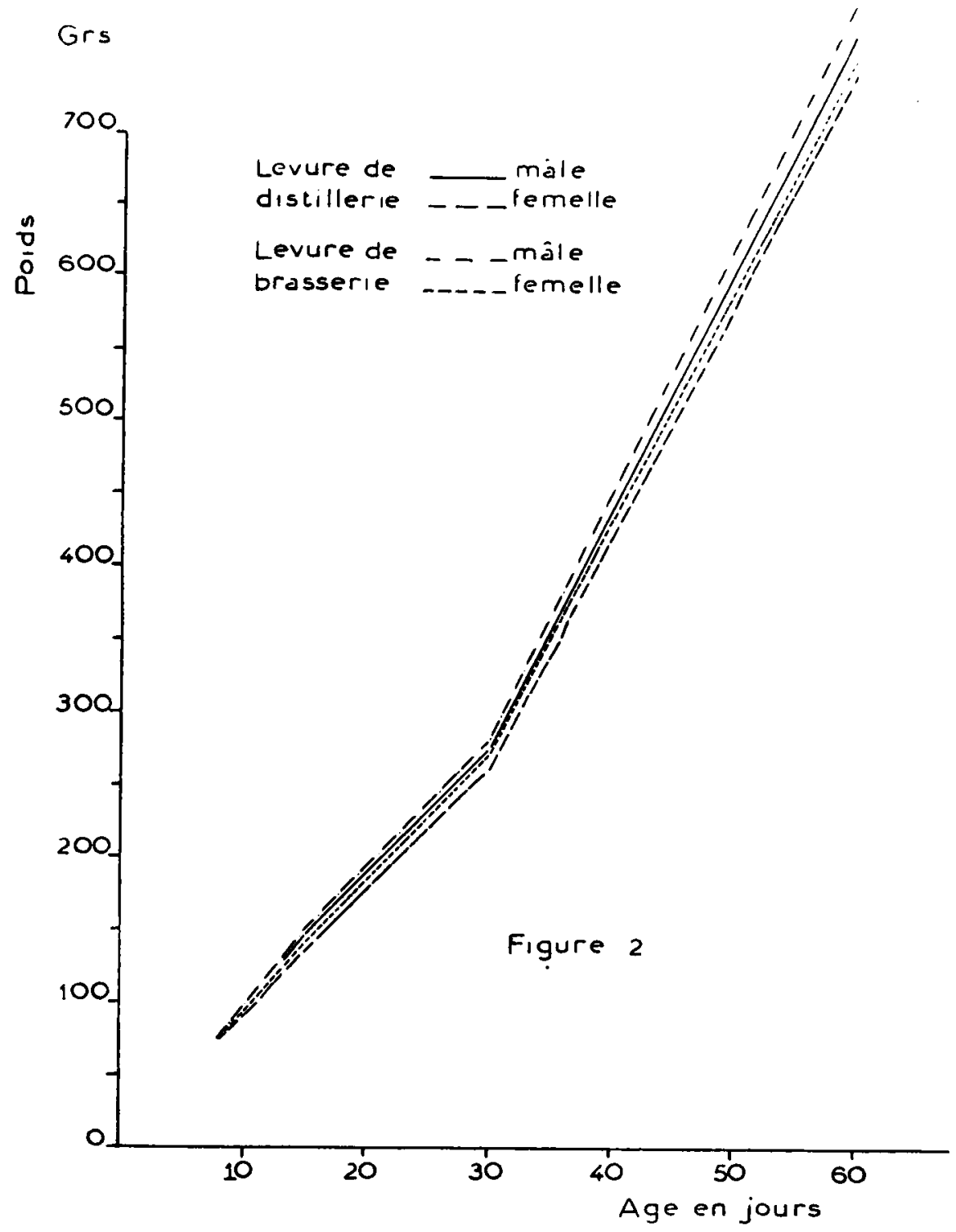

distillerie et la levure de brasserie ont la même valeur pour la croissance des poussins.

Notons que la levure de distillerie utilisée avait la même provenance que celles employées dans les précédentes expériences. 


\section{Conclusions générales}

I $^{\circ}$ La levure de distillerie peut s'utiliser sans inconvénient en quantités importantes dans l'alimentation des poules pondeuses.

Nous avons utilisé cet aliment, à la dose de 9,25 p. Ioo dans la ration totale des volailles, en l'absence de tout autre aliment azoté d'origine animale. Nous n'avons constaté aucune différence de comportement entre les animaux ainsi nourris et ceux d'un lot témoin recevant un régime normal équiazoté enrichi en farine de viande.

La ponte et les phénomènes de reproduction dans les deux lots ont été identiques.

$2^{\circ}$ La levure de distillerie introduite dans un régime complet pour poussins à la dose de 14 p. Ioo a donné des résultats égaux - quant à la croissance des poulettes - ou supérieurs - quant à la croissance des mâles - à un régime témoin équi-azoté contenant des farines de viande, de poisson, et de sang.

L'emplumage s'est effectué de façon plus rapide dans le lot expérimental.

Les poulettes soumises à ce régime sont entrées normalement en ponte vers 6 mois et demi.

$3^{\circ}$ Les levures de distillerie et de brasserie introduites à la dose de Io p. Ioo dans un régime pour poussins ont donné des résultats de croissance identiques.

$4^{\circ}$ Les Industries Françaises ne mettent malheureusement pas sur le marché un produit de qualité constante. Les conclusions de ce travail ne valent que pour les qualités de levure que nous avons utilisées ${ }^{1}$ ).

\section{BIBLIOGRAPHIL:}

(1) Agricullural Research in Neve IIampshire, 44, Bull. 372, 1947.

(2) I'rogress Report : Yeast culture investigations : live yeast culture tested for production efficiency as feedstuff for chicks: California Agriculture, 3, 7, 3, I949.

(3) Progress Report : Yeast culture, investigations: California Agriculture, 4, e3, 1950.

(4) Campbell (P. A.) and Ringrose (R. C.).-Comparison of torula yeast and brewers'yeast for poultry feeding; lieedstuffs, 22, I 7, 32, I 950 .

(5) Grnnis (M. C.). - Utilisation of yeast in chicken and turkey diets : Feedstuffs, 21, I4, 42,1949 .

(6) Kennard (D. C.), Chamberian (V. P.). - (Ohio Exp. St. Bull., i 948.

(7) Milligan (J. L.), Anjerson (J. O.), Combs (G. F.), Briggs (G. H.). - Dried pénicillin mycellium as a riboflavin source in chick rations : Poultry Sci, 29, 6, 870, $195^{\circ}$.

(8) Marco (J.) et Lahaye (J.). - Traití complet d'Aviculture. Librairie Agricole de la Maison Rustique, Paris.

(9) Novak (A. F.), Hauge (S. M.), Carkik (C. W.). -- An unidentified growth factor in distillers'dried solubles essential for chicks: Poullry Sci., 26, 6, 604, 1947.

(io) Synoli) (R. E.), Carrik (C. W.), Roberts (R. E.), Havie (S. M.). - Distillers' dried solubles as vitamin supplement in chick ration : P'ouliry Sci., 22, 4, 323, I943.

(I I) Titus (H. W.). - Food and life: Yearbook of Agriculture I 929 ou U.S. Département of agriculture U.S. Gouvernement Printing Office, p. 819-844.

(1 2) WaLlis (G. C.). - Yeast and yeast products in animal feeding : lieedstuffs, 22, 29, 44, $195^{\circ}$.

$\left(^{1}\right)$ La levure utilisée a été fournie par la distillerie de Provin; (S. et M.) elle avait été séchée à haute température. 